\title{
Modification of a proto-lunar disk by hydrodynamic escape of silicate vapor
}

\author{
Hidenori Genda and Yutaka Abe \\ Department of Earth and Planetary Science, The University of Tokyo, 7-3-1 Hongo, Bunkyo-ku, Tokyo 113-0033, Japan
}

(Received April 3, 2002; Revised November 27, 2002; Accepted January 17, 2003)

\begin{abstract}
We have estimated a criterion for escape of silicate vapor from the disk formed by a giant impact. Escape from the disk affects the mass distribution and specific angular momentum of the disk. We applied the results to the disk formed by the Moon-forming impact. In the case of a hot ( $>6000 \mathrm{~K})$ and highly vaporized ( $>63 \%)$ disk, the material outside of the Roche radius escapes. This indicates that the formation of the Moon is influenced by the thermal state of the disk just after the giant impact.
\end{abstract}

\section{Introduction}

According to the giant impact hypothesis, a circumterrestrial disk is generated by an oblique impact of a Mars-sized protoplanet onto the proto-Earth (e.g., Benz et al., 1989; Cameron and Benz, 1991; Cameron, 1997; Canup and Asphaug, 2001). The disk just after the Moon-forming impact is very hot and partially vaporized. Release of latent heat due to condensation of vaporized silicate results in a nearly isothermal disk. Since an isothermal gas is not bounded by the gravity of the Earth, silicate vapor possibly escapes from the disk through a hydrodynamic outflow. Escape from the disk affects the formation of the Moon by changing the mass and shape of the disk. In addition, if the vaporized gas component of the disk is selectively lost, for example, due to the sedimentation of the condensate liquid drops toward the central plane of the disk (Machida and Abe, 2002), the disk is enriched in refractory components. This will affect not only the Moon's composition, but also the Earth's composition.

In this paper, we discuss the effect of disk escape on the mass distribution in the disk rather than the chemical effect. First, we show that the adiabat of partially vaporized silicate is very close to isothermal (Section 2). Then we analytically estimate a criterion for the disk escape and its dependence on the physical parameters of the disk (Section 3). Finally, we discuss the effect on Moon formation.

\section{Thermal Structure of an Impact-Generated Disk}

We assume the proto-lunar disk is isentropic. If the disk is well-mixed in its formation and/or every disk's material experiences the uniform level of the shock compression by the impact, the disk's structure becomes isentropic. According to the recent SPH simulations (e.g., Canup and Asphaug, 2001; Cameron, 1997), since the major part of the disk material derives from the impactor's hemisphere against the impact point and the vicinity of its surface, it is thought that the disk material experiences the nearly uniform level of the

Copy right(c) The Society of Geomagnetism and Earth, Planetary and Space Sciences (SGEPSS); The Seismological Society of Japan; The Volcanological Society of Japan; The Geodetic Society of Japan; The Japanese Society for Planetary Sciences. shock compression. Here, we calculate the thermal structure of a proto-lunar disk, that is, the adiabat of the disk material. We shows the adiabat of the disk material is very close to isothermal due to the very large latent heat for condensation of silicate vapor, and is nicely approximated by a polytrope with exponent 1.05 .

For simplicity, we assume that the disk material is one component and two phases. When $X$ is defined as the mass fraction of vapor in the disk, the pressure in the disk is given by the ideal gas law;

$$
p V=X \frac{R T}{M_{m}}
$$

where $p, V, T, R$ and $M_{m}$ are the pressure, the specific volume, the temperature, the gas constant, and the molecular weight of vapor, respectively. The assumption of the ideal gas law is valid, because the disk's density of silicate vapor (typically, $<10 \mathrm{~kg} / \mathrm{m}^{3}$ ) is much less than the density of silicate melt $\left(\sim 3500 \mathrm{~kg} / \mathrm{m}^{3}\right)$. The equations of entropy change and vapor-condensate equilibrium (ClausiusClapeyron equation) are given as follows:

$$
\begin{gathered}
c_{p}^{\prime} \frac{d T}{T}-\frac{R}{M_{m}} \frac{d p}{p}-d\left[\frac{L(1-X)}{T}\right]=d S, \\
\frac{d p}{d T}=\frac{M_{m} L p}{R T^{2}},
\end{gathered}
$$

where $c_{p}^{\prime}$ is the isopiestic specific heat of vapor-condensate mixture, which is given by $c_{p}^{\prime}=X c_{p}+(1-X) C_{p}$ with the isopiestic specific heats of vapor, $c_{p}$, and condensate (liquid), $C_{p} . L$ and $d S$ are the latent heat for vaporization, and entropy change, respectively.

When we assume constant $M_{m}$, the total differentiation of Eq. (1) is written as

$$
V d p+p d V=\frac{R}{M_{m}}(X d T+T d X) .
$$

Using the specific heat ratio of the vapor phase, $\gamma_{g}$, we have $R / M_{m}=c_{p}\left(\gamma_{g}-1\right) / \gamma_{g}$. Hence,

$$
V d p+p d V=c_{p} \gamma_{g}^{\prime}(X d T+T d X),
$$




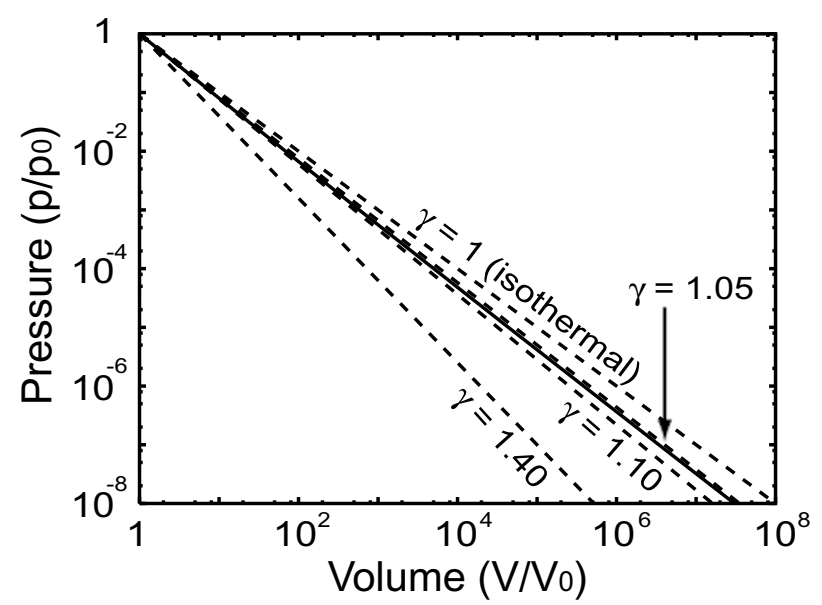

Fig. 1. Adiabatic change for one component and two phases. The solid curve represents adiabatic change of partially vaporized silicate for a typical value $\left(L=2.0 \times 10^{7} \mathrm{~J} / \mathrm{kg}\right.$, e.g., Hashimoto $(1983), c_{p}=10^{3} \mathrm{~J} / \mathrm{kgK}$ and $\left.\gamma_{g}=1.4\right)$. The mass fraction of vapor $\left(X_{0}\right)$ and the temperature at the standard volume $\left(V_{0}\right)$ are 0.5 and $5000 \mathrm{~K}$, respectively. Both axes are normalized by the reference physical value. Dashed curves represent adiabatic change of polytropic gas in the cases of $\gamma=1$ (isothermal), 1.05 , 1.10 and 1.40

where $\gamma_{g}^{\prime}=\left(\gamma_{g}-1\right) / \gamma_{g}$. When we assume an adiabatic process $(d S=0)$ and the constant latent heat $(d L=0)$, Eq. (2) is written as

$$
\left[c_{p}^{\prime}+\frac{L(1-X)}{T}\right] \frac{d T}{T}-\frac{R}{M_{m}} \frac{d p}{p}+\frac{L}{T} d X=0 .
$$

From Eqs. (3), (5), and (6), we can obtain $d X / d V, d p / d V$ and $d T / d V$ as follows:

$$
\begin{gathered}
\frac{d X}{d V}=\gamma_{g}^{\prime} \cdot \frac{X}{V}\left(\frac{\frac{L}{c_{p} T}-\gamma_{g}^{\prime}}{\frac{c_{p}^{\prime} T}{L X}-1}+\gamma_{g}^{\prime}\right)^{-1}, \\
\frac{d p}{d V}=-\frac{p}{V}\left[1-\gamma_{g}^{\prime} \frac{c_{p} T}{L}\left(2-\frac{c_{p}^{\prime} T}{L X}\right)\right]^{-1}, \\
\frac{d T}{d V}=-\gamma_{g}^{\prime} \frac{c_{p} T}{L} \times \frac{T}{V}\left[1-\gamma_{g}^{\prime} \frac{c_{p} T}{L}\left(2-\frac{c_{p}^{\prime} T}{L X}\right)\right]^{-1} .
\end{gathered}
$$

In reality, the molecular weight is not constant in high temperature silicate vapor. However, owing to very large latent heat, the resulted adiabat is not sensitive to the change of molecular weight. Hence, we assumed a constant molecular weight here.

We can numerically obtain a relation between $p$ and $V$ from Eq. (7) to (9). Figure 1 shows the resulting adiabat for a typical value of partially vaporized silicate (see figure caption). The adiabat can be approximated by a polytrope $\left(p \propto V^{-\gamma}\right)$ with polytropic exponent $\gamma$ nearly 1.05 . Since $\gamma=1$ means isothermal, the adiabat of partially vaporized silicate is likely very close to isothermal. This isothermal characteristic is due to very large latent heat for condensation of silicate vapor.

\section{A Criterion for the Escape}

First of all, we derive a criterion for the break down of hydrostatic equilibrium in the case of the one-dimensional spherical polytropic atmosphere. When such a criterion is satisfied, it is known that the hydrodynamic escape occurs just like solar wind (Parker, 1963). Next, we derive a general criterion for the break down of hydrostatic equilibrium in the case of an arbitrary two-dimensional axisymmetric polytropic disk, and compare with the case of the spherical atmosphere. Next, we assume the disk with a power law distribution of the pressure at the central plane of the disk, and derive the criterion for the escape.

When we take the distance from the planetary center as $x$, the hydrostatic equation of the spherically symmetric atmosphere is written as

$$
\frac{1}{\rho} \frac{d p}{d x}+\frac{G M}{x^{2}}=0
$$

where $\rho, G$ and $M$ are the density of the atmosphere, the gravitational constant, and the mass of the Earth, respectively. We assume the polytropic atmosphere. Using the polytropic exponent, $\gamma$, we get the following relation between the pressure and the density:

$$
\frac{p}{p_{0}}=\left(\frac{\rho}{\rho_{0}}\right)^{\gamma}
$$

where $p_{0}$ and $\rho_{0}$ are the pressure and density at the reference point. Here, we take the reference point as the planetary surface. Using Eq. (11) we can solve Eq. (10) and get the distribution of the atmospheric pressure as follows:

$$
\frac{p}{p_{0}}=\left[\frac{\gamma-1}{\gamma} \lambda_{0}\left(\frac{r_{0}}{x}-1\right)+1\right]^{\frac{\gamma}{\gamma-1}},
$$

where $r_{0}$ is the planetary radius and $\lambda_{0}$ is the escape parameter, which is defined by

$$
\lambda_{0}=\frac{G M \rho_{0}}{r_{0} p_{0}} .
$$

Using the ideal gas law, Eq. (1), we obtain $\lambda_{0}=$ $G M M_{m} /\left(X_{0} R T_{0} r_{0}\right)$ from Eq. (13). Therefore, the escape parameter turns out to be the ratio of the gravitational energy required for escape from the Earth to the thermal energy of the atmosphere.

For the atmosphere bounded by the Earth's gravity, the atmospheric pressure should be zero at a certain distance. An atmosphere with finite pressure at infinite distance can be hydrostatically equilibrated, only when the finite pressure is balanced with the ambient pressure in the distant space. Otherwise, it expands toward the space and lost. This is the break down of the hydrostatic equilibrium and the onset of the hydrodynamic escape. The criterion of the onset of the hydrodynamic escape is given by the case when the pressure is zero at $x \rightarrow \infty$ in Eq. (12);

$$
\lambda_{0}<\frac{\gamma}{\gamma-1} .
$$

It is noted that both near-isothermal and hot atmospheres are difficult to be in hydrostatic equilibrium.

Next, we derive a general criterion for the break down of hydrostatic equilibrium in the case of the two-dimensional axisymmetric disk. When we take the radial direction of the 
disk as $r$, and the vertical direction as $z$, the equations for the hydrostatic equilibrium of the disk are written as

$$
\begin{gathered}
\frac{1}{\rho} \frac{d p}{d r}+G M \frac{r}{\left(r^{2}+z^{2}\right)^{\frac{3}{2}}}-r \omega^{2}=0, \\
\frac{1}{\rho} \frac{d p}{d z}+G M \frac{z}{\left(r^{2}+z^{2}\right)^{\frac{3}{2}}}=0,
\end{gathered}
$$

where $\omega$ is the angular velocity of disk material. Let's consider the force balance in $r$-direction first. Eq. (15) can be rewritten as,

$$
\omega^{2}=\frac{1}{r}\left[G M \frac{r}{\left(r^{2}+z^{2}\right)^{\frac{3}{2}}}+\frac{1}{\rho} \frac{d p}{d r}\right] .
$$

If the pressure decreases so steeply with distance, the right side of Eq. (17) gets negative. However, the left side of Eq. (17) must be positive. Therefore, there is a constraint for the pressure gradient, that is,

$$
\frac{d p}{d r}>-\frac{G M \rho r}{\left(r^{2}+z^{2}\right)^{\frac{3}{2}}} .
$$

This is a necessary condition for the existence of a rotating disk. As far as this condition is satisfied, we can satisfy the force balance in $r$-direction (Eq. (15)) for arbitrarily given radial distribution of pressure by choosing the angular velocity.

On the other hand, Eq. (16), which gives us the disk's structure of $z$-direction, gives the condition for the onset of hydrodynamic outflow. We assume the polytropic structure of $z$-direction and take the reference point as the central plane of the disk. Thus, we get the following relation between the pressure and the density:

$$
\frac{p}{p_{r, 0}}=\left(\frac{\rho}{\rho_{r, 0}}\right)^{\gamma}
$$

where $\rho_{r, 0}$ and $p_{r, 0}$ are the density and pressure at the central plane of the disk, respectively, at the distance $r$. Using Eq. (19), we can solve Eq. (16), and the distribution of the pressure and density are given by

$$
\begin{aligned}
\frac{p}{p_{r, 0}} & =\left[\frac{\gamma-1}{\gamma} \lambda_{r, 0}\left(\frac{r}{\sqrt{r^{2}+z^{2}}}-1\right)+1\right]^{\frac{\gamma}{\gamma-1}}, \\
\frac{\rho}{\rho_{r, 0}} & =\left[\frac{\gamma-1}{\gamma} \lambda_{r, 0}\left(\frac{r}{\sqrt{r^{2}+z^{2}}}-1\right)+1\right]^{\frac{1}{\gamma-1}},
\end{aligned}
$$

where $\lambda_{r, 0}$ is the local escape parameter at the central plane, and defined by

$$
\lambda_{r, 0}=\frac{G M \rho_{r, 0}}{r p_{r, 0}} .
$$

Like a spherically symmetric atmosphere, the pressure of the bounded disk is expected to be zero at a certain distance from the Earth. Therefore, the criterion for the break down of the hydrostatic equilibrium is obtained from Eq. (20) or (21) with $z \rightarrow \infty$ :

$$
\lambda_{r, 0}<\frac{\gamma}{\gamma-1}
$$

This criterion very closely resembles the one for the spherical atmosphere (Eq. (14)). If the local escape parameter decreases with radial distance, we will find the critical radial distance over which the disk is not in a hydrostatic equilibrium. As can be shown from Eq. (23), an isothermal disk (i.e., $\gamma=1$ ) cannot be in hydrostatic equilibrium at any distance from the planet.

Even if we consider an adiabatic disk, the radial distribution of the local escape parameter is not uniquely determined, because the radial distribution of pressure is not uniquely constrained by the hydrostatic equilibrium as noted before. In the following we consider a specific case in which the radial distribution of pressure at the central plane of the disk $\left(p_{r, 0}\right)$ is proportional to a power law of the radial distance:

$$
p_{r, 0}=p_{0,0}\left(\frac{r}{r_{0}}\right)^{-\zeta}
$$

where $\zeta$ is the parameter that represents a radial distribution of pressure, and $p_{0,0}$ is the pressure at the disk's central plane and the planetary surface. We newly take the reference point on the planetary surface at the central plane. The disk becomes more compact with increasing $\zeta$ value. In reality, the value of $\zeta$ is determined through the impact process, which depends on the impact parameter and the equation of state of the silicate, and so on. Here, we put $\zeta$ as a parameter.

Since we consider an isentropic disk, the density distribution of the central plane is given from Eq. (24);

$$
\rho_{r, 0}=\rho_{0,0}\left(\frac{r}{r_{0}}\right)^{-\frac{\zeta}{\gamma}}
$$

where $\rho_{0,0}$ is the density at the reference point. Using Eqs. (20), (22), (24) and (25), we obtain the pressure distribution of the disk as follows;

$$
\frac{p}{p_{0,0}}=\left[\frac{\gamma-1}{\gamma} \lambda_{0,0}\left(\frac{r_{0}}{\sqrt{r^{2}+z^{2}}}-\frac{r_{0}}{r}\right)+\left(\frac{r_{0}}{r}\right)^{\zeta \frac{\gamma-1}{\gamma}}\right]^{\frac{\gamma}{\gamma-1}},
$$

and in similar way, the density distribution of the disk is obtained by

$$
\frac{\rho}{\rho_{0,0}}=\left[\frac{\gamma-1}{\gamma} \lambda_{0,0}\left(\frac{r_{0}}{\sqrt{r^{2}+z^{2}}}-\frac{r_{0}}{r}\right)+\left(\frac{r_{0}}{r}\right)^{\zeta \frac{\gamma-1}{\gamma}}\right]^{\frac{1}{\gamma-1}},
$$

where $\lambda_{0,0}$ is the escape parameter at the reference point and defined by

$$
\lambda_{0,0}=\frac{G M \rho_{0,0}}{r_{0} p_{0,0}} .
$$

Using the ideal gas law, Eq. (1), we also obtain $\lambda_{0,0}=$ $G M M_{m} /\left(X_{0,0} R T_{0,0} r_{0}\right)$, where $X_{0,0}$ and $T_{0,0}$ are the mass fraction of vapor and the temperature at the reference point, respectively. Substituting Eqs. (26) and (27) into Eq. (17), we also obtain the angular velocity distribution as follows;

$$
\omega^{2}=\frac{G M}{r^{3}}\left[1-\frac{\zeta}{\lambda_{0,0}}\left(\frac{r_{0}}{r}\right)^{\zeta \frac{\gamma-1}{\gamma}-1}\right] .
$$




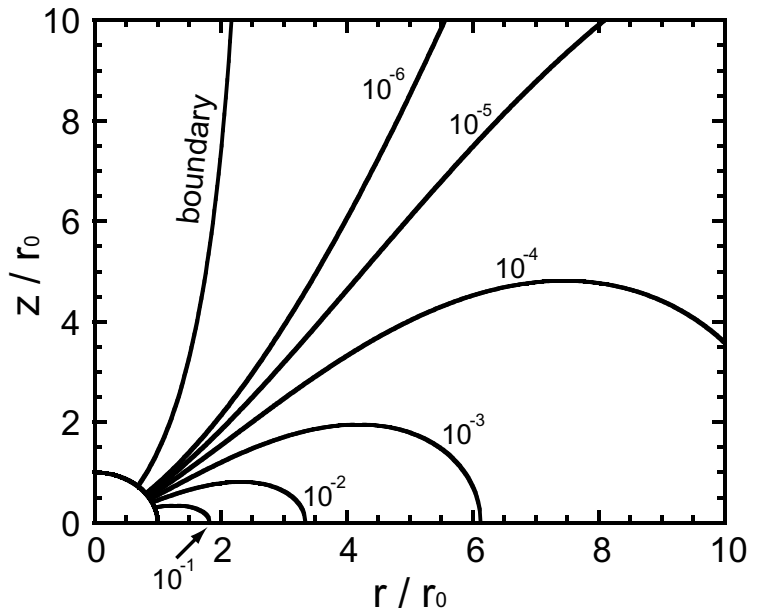

Fig. 2. Density distribution in a disk that cannot keep at hydrostatic equilibrium $\left(\zeta=4, \gamma=1.05, \lambda_{0,0}=50\right)$. It should be noted that the disk material is close to isothermal. Density is normalized by the value at $r=r_{0}, z=0$, that is $\rho_{0,0}$. The 'boundary' in the figure indicates surface of the zero density. Outside of a certain radius, the disk has finite density at $z \rightarrow \infty$, that is, the disk material outside of the disk is not bounded by the gravity of the Earth.

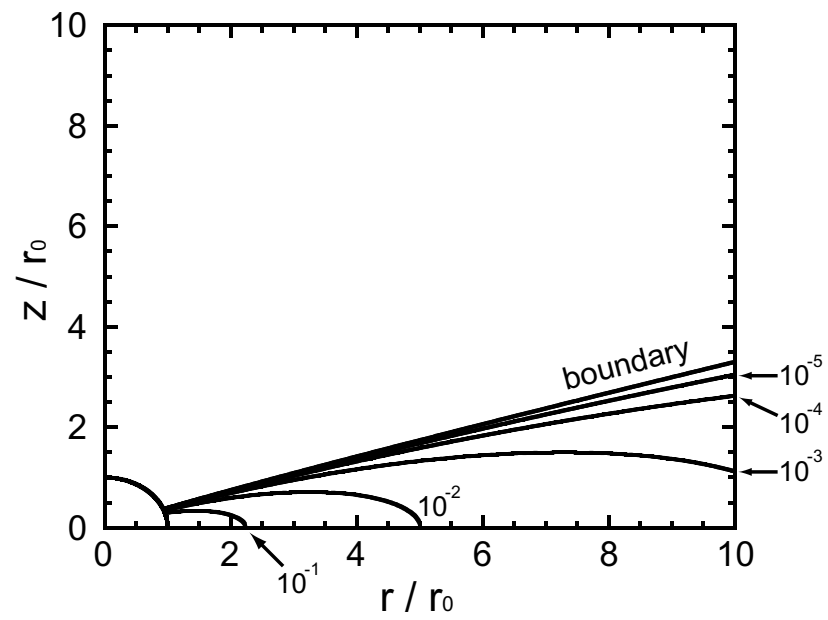

Fig. 3. Density distribution in a disk at hydrostatic equilibrium $(\zeta=4$, $\left.\gamma=1.4, \lambda_{0,0}=50\right)$. Density is normalized by the value at $r=r_{0}$, $z=0$, that is $\rho_{0,0}$. The 'boundary' in the figure indicates surface of the zero density. The disk has zero density at $z \rightarrow \infty$, that is, the disk material is tightly bounded by the gravity of the Earth.

Since the right side of this equation must be positive, we obtain the relation of $\zeta \leq \lambda_{0,0}$. Thus, the rotating power law disk exists only when $\zeta \leq \lambda_{0,0}$ is satisfied. This means the maximum $\zeta$ is limited depending on the disk's temperature. In other words, a hot disk cannot be compact.

Substituting Eqs. (24) and (25) into Eq. (22), we can obtain the local escape parameter, $\lambda_{r, 0}$, as follows;

$$
\lambda_{r, 0}=\lambda_{0,0}\left(\frac{r}{r_{0}}\right)^{\frac{\gamma-1}{\gamma} \zeta-1}
$$

From Eq. (30), $\lambda_{r, 0}$ decreases with increasing the radial distance, when the following condition is satisfied;

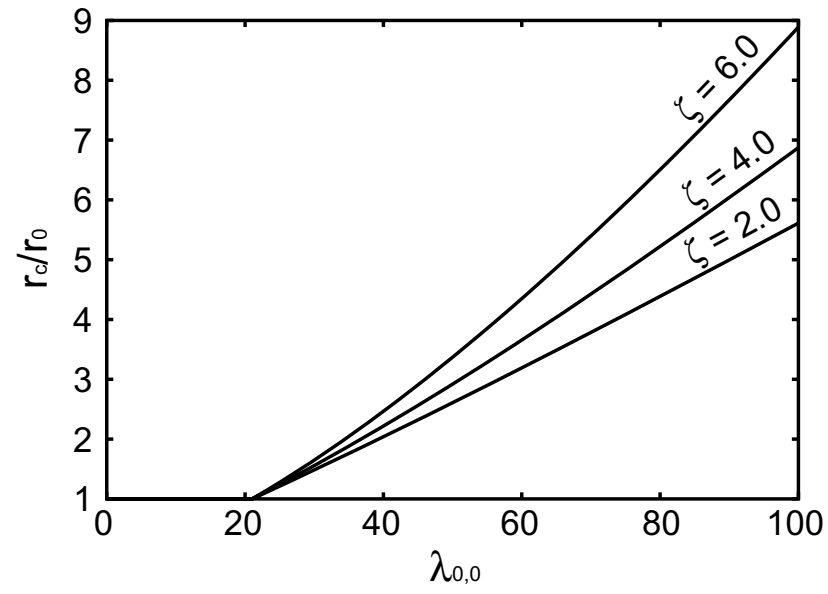

Fig. 4. Dependence of critical radius $\left(r_{c}\right)$ on $\lambda_{0,0}$, and $\zeta$ for $\gamma=1.05$. Since $r_{c} \leq r_{0}$ in the region of $\lambda_{0,0} \leq 21$, escape occurs directly from the planetary surface.

$$
\zeta<\frac{\gamma}{\gamma-1}
$$

Such a disk has a certain critical radius over which Eq. (23) is satisfied, and cannot keep in a hydrostatic equilibrium. Eq. (31) indicates both near-isothermal and broad disk has a portion of escaping region. We define the critical radius as $r_{c}$. From Eq. (23) and (30), the critical radius is given by

$$
\frac{r_{c}}{r_{0}}=\left(\frac{\gamma-1}{\gamma} \lambda_{0,0}\right)^{-\frac{\gamma}{\zeta(\gamma-1)-\gamma}} .
$$

Figure 2 shows the density distribution for the disk with $\zeta=4, \gamma=1.05$ and $\lambda_{0,0}=50$, that is, the disk is satisfying Eq. (31). The disk has finite density at $z \rightarrow \infty$ outside of about $3 r_{0}$. Therefore, the disk cannot remain in hydrostatic equilibrium and escape from the outer part of the disk is expected. On the other hand, Fig. 3 shows the density distribution for the disk with $\zeta=4, \gamma=1.4$ and $\lambda_{0,0}=$ 50 , that is, the disk is not satisfying Eq. (31), and the disk has zero density boundary everywhere in the $z$-direction. Therefore, the disk can keep in hydrostatic equilibrium and be tightly bounded by the gravity of the Earth. Figure 4 shows the dependence of the critical radius, $r_{c}$, on $\zeta$ and $\lambda_{0,0}$ for $\gamma=1.05$. The critical radius becomes small with decreasing $\lambda_{0,0}$ value, that is, escape from inner part of the disk occurs. It can be seen that $r_{c}$ decreases slightly with decreasing $\zeta$ value. When $\lambda_{0,0}<\gamma /(\gamma-1), r_{c}$ is less than $r_{0}$, and escape occurs from the planetary surface.

\section{Discussion and Implication}

We have analytically derived the criterion for the break down of hydrostatic equilibrium, and shown that a nearly isothermal disk cannot keep a state in the hydrostatic equilibrium. In the case of an isentropic disk, the disk material outside of the critical radius $\left(r_{c}\right)$ is lost by hydrodynamic outflow. In reality, cooling of the disk will retard the escape, because $r_{c}$ becomes large due to decreasing temperature. However, we ignored the radiative cooling and assumed adiabatic expansion, because the radiative cooling of the disk is inefficient as the disk is massive ( $\sim$ the present lunar mass) and 


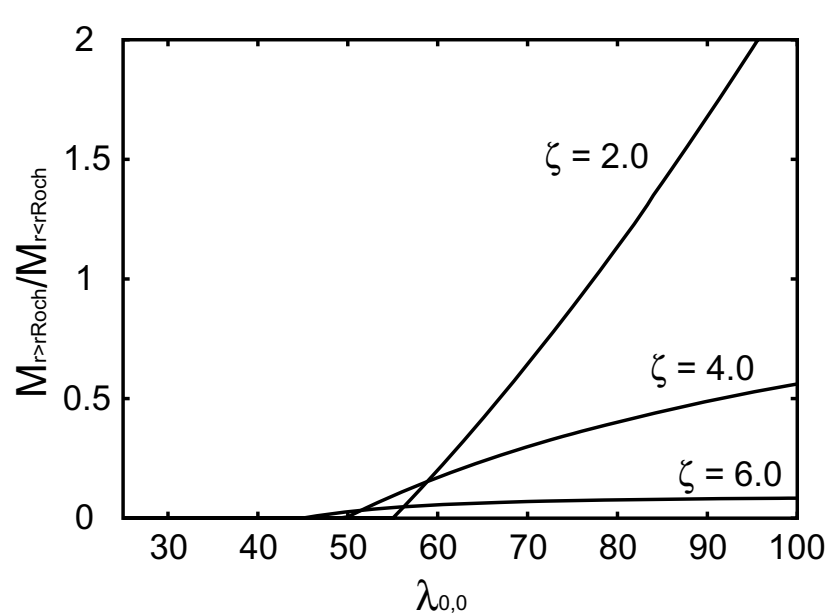

Fig. 5. The mass outside the Roche radius for $\gamma=1.05$, on the assumption that the mass outside of critical radius is lost by escape. The mass is normalized by the mass inside the Roche radius.

is optically thick due to dust particles produced by condensation of silicate vapor. Therefore, material outside of the critical radius will expand adiabatically. Thus, we assume disk material outside of the critical radius is lost.

We apply the obtained results to the proto-lunar disk. When we assume that the disk material outside of the critical radius is completely lost, the mass outside of the Roche radius $\left(r_{\text {Roche }} \sim 2.9 r_{0}\right)$ is shown in Fig. 5 for $\gamma=1.05$. When $\lambda_{0,0} \leq 60$, almost no material remains outside of the Roche radius irrespective of initial mass distribution. From Eq. (28), we obtain $X_{0,0} \geq 0.63$ to satisfy $\lambda_{0,0} \leq 60$, using $M=6.0 \times 10^{24} \mathrm{~kg}$ (the present Earth's mass), $r_{0}=$ $6.4 \times 10^{6} \mathrm{~m}$ (the present Earth's radius), $M_{m}=30 \times 10^{-3}$ $\mathrm{kg} / \mathrm{mol}$ and $T_{0,0}=6000 \mathrm{~K}$ (e.g., Cameron, 1997). Therefore, in the case of the highly vaporized disk $\left(X_{0,0} \geq 0.63\right)$, even if the Moon-forming impact scatters sufficiently a large amount of disk materials beyond the Roche radius, almost all the material outside of the Roche radius is lost by escape.

Moon accretion cannot occur within the Roche radius, because the tidal force exceeds the self-gravity of the disk materials. If the Moon-forming impact scatters a sufficiently large amount of disk materials beyond the Roche radius, direct accretion of a Moon from such materials is possible. Cameron and Benz (1991) exploit this possibility. However, our results rule out such possibility for $X_{0,0} \geq 0.63$. An alternative view is more likely, that is, accretion of the Moon after spreading of an initially compact disk owing to angular momentum transfer following the occurrence of gravitational instability in the disk (Ida et al., 1997).

The specific angular momentum of the disk is very important parameter in determining the mass of the finally formed Moon $\left(M_{\text {moon }}\right)$. According to Ida et al. (1997), its relation is numerically obtained as follows;

$$
\frac{M_{\text {moon }}}{M_{\text {disk }}} \approx \frac{1.9}{\sqrt{G M r_{\text {Roche }}}} \frac{J_{\text {disk }}}{M_{\text {disk }}}-1.15,
$$

where $M_{\text {disk }}$ and $J_{\text {disk }}$ are the mass and angular momentum of the disk, and $r_{\text {Roche }}$ is the Roche radius. When no escape occurs, that is, $\lambda_{0,0} \rightarrow \infty, J_{\text {disk }} / M_{\text {disk }}$ strongly depends on the disk's initial mass distribution (corresponding to $\zeta$ ) and

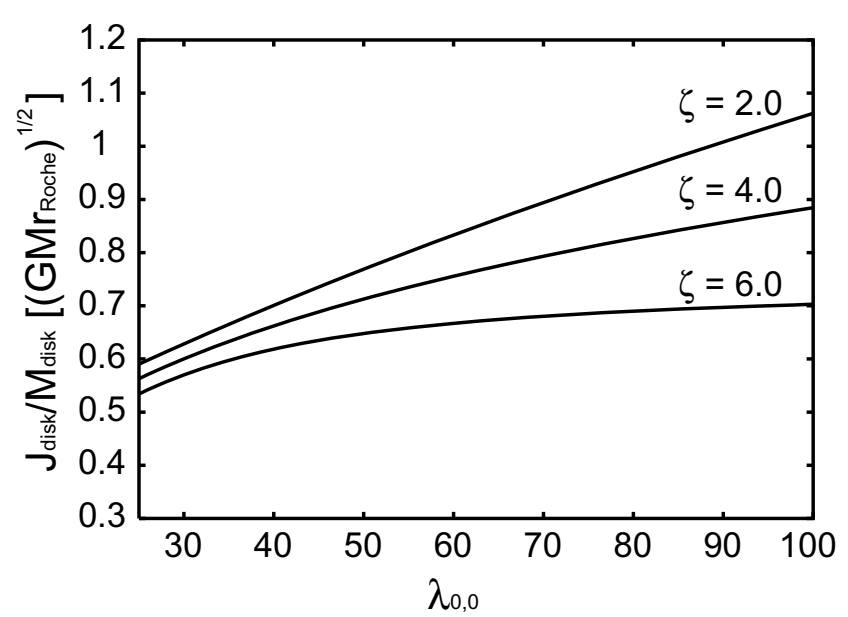

Fig. 6. Dependence of $\lambda_{0,0}$ on the specific angular momentum of the disk for $\gamma=1.05$, on the assumption that the mass outside of the critical radius is lost by escape.

the position of the disk's outer edge $\left(r_{\text {edge }}\right)$, which are determined by the physical process of the impact. For example, when $r_{\text {edge }}=9 r_{0}, J_{\text {disk }} / M_{\text {disk }}$ is $1.36,0.99$ and 0.74 (normalized by $\sqrt{G M r_{\text {Roche }}}$ ) for $\zeta=2.0,4.0$ and 6.0 , respectively. On the other hand, when escape occurs, $J_{\text {disk }} / M_{\text {disk }}$ decreases, because the outer part of the disk, which is lost by escape, has larger specific angular momentum than the inner part. Figure 6 shows the specific angular momentum $\left(J_{\text {disk }} / M_{\text {disk }}\right)$ of the disk, whose mass outside of the critical radius is lost by escape. $J_{\text {disk }} / M_{\text {disk }}$ is insensitive to $\zeta$ for small $\lambda_{0,0}$ values and is smaller than $0.8 \sqrt{G M r_{\text {Roche }}}$ at $\lambda_{0,0}<60$. This implies $M_{\text {moon }} / M_{\text {disk }}<0.37$. Thus, the Moon formation process is significantly affected by the temperature and vapor mass fraction of the disk as well as the dynamic parameters $\zeta$ and $r_{\text {edge }}$.

Acknowledgments. The authors thank S. Ida, S. Sasaki, and S. Watanabe for helpful discussions and comments. We also thank K. Zahnle and K. Kuramoto for their constructive review. This study was completed under the support of JSPS Research Fellowship.

\section{References}

Benz, W., A. G. W. Cameron, and H. J. Melosh, The origin of the Moon and the single-impact hypothesis III, Icarus, 81, 113-131, 1989.

Cameron, A. G. W., The origin of the Moon and the single-impact hypothesis V, Icarus, 126, 126-137, 1997.

Cameron, A. G. W. and W. Benz, The origin of the Moon and the singleimpact hypothesis IV, Icarus, 92, 204-216, 1991.

Canup, R. M. and E. Asphaug, Origin of the Moon in a giant impact near the end of the Earth's formation, Nature, 412, 708-712, 2001.

Hashimoto, A., Evaporation metamorphism in the early solar nebulaevaporation experiments on the melt $\mathrm{FeO}-\mathrm{MgO}-\mathrm{SiO}_{2}-\mathrm{CaO}-\mathrm{Al}_{2} \mathrm{O}_{3}$ and chemical fractionations of primitive materials, Geochem. J., 17, 111-145, 1983.

Ida, S., R. M. Canup, and G. R. Stewart, Lunar accretion from an impactgenerated disk, Nature, 389, 353-357, 1997.

Machida, R. and Y. Abe, Evolution of the proto-lunar disk: instability of the dust layer and the moon forming material, Lunar Planet. Sci. Conf., XXXIII, Abstract \#1690 in CD-ROM, 2002.

Parker, E. N., Hydrostatic properties of a coronal atmosphere, in Interplanetary Dynamical Processes, Interscience Publishers, New York, 1963.

H. Genda (e-mail: genda@sys.eps.s.u-tokyo.ac.jp) and Y. Abe 